\title{
LA NUEVA NORMATIVA ITALIANA EN MATERIA DE OBRAS HUÉRFANAS
}

\author{
Sofía Rodríguez Moreno*
}

\section{INTRODUCCIÓN}

El Decreto legislativo 163 del 10 de noviembre de 2014 ha introducido en el ordenamiento jurídico italiano las normas de trasposición de la Directiva europea 2012/28/Ue. Dicho decreto modifica la ley italiana de derechos de autor (Ley 633 de 1941) incorporando los artículos 69 bis a 69 septies con el fin de consentir algunas utilizaciones de las obras huérfanas que de lo contrario no estarían permitidas por el derecho de autor.

La atención al fenómeno de las obras huérfanas u obras protegidas por el derecho de autor y las prestaciones amparadas por los derechos conexos ${ }^{1}$ cuyos

\footnotetext{
* Abogada en Colombia e Italia. Actualmente asesora empresas colombianas e italianas en materia de contratos comerciales, propiedad intelectual, derecho societario y civil. Reside en Milán. Doctora en Derecho Privado de la Università degli Studi di Pavia (Italia). Correo-e: avv.sofiarodriguez@gmail.com Recibido: 2 de octubre de 2015. Aceptado: 29 de octubre de 2015. Este artículo es una reelaboración del documento publicado en italiano en la revista científica Le Nuove Leggi Civili Commentate (NLCC): Rodríguez-Moreno, Sofía. La nuova disciplina delle opere orfane NLCC, 2015, pp. 893-911. Para citar el artículo: S. Rodríguez M. La nueva normatividad italiana en materia de obras huérfanas. Revista la Propiedad Inmaterial n. ${ }^{\circ}$ 20, Universidad Externado de Colombia, julio-diciembre 2015 , pp. 135-151. DOI: http://dx.doi.org/10.18601/16571959.n20.07

1. El problema de las obras huérfanas no se limita a las obras protegidas por el derecho de autor sino que comprende además las prestaciones intelectuales amparadas por los derechos conexos. La doctrina, los documentos oficiales y los estudios realizados por instituciones internacionales sobre la materia utilizan indistintamente la expresión "obra huérfana" para referirse tanto a las obras protegidas por el derecho de autor como a las prestaciones intelectuales tuteladas por los derechos conexos. Cfr. Ivir, Recasting of Copyright and Related Rights for the Knowledge Economy, noviembre 2006, en http://www.ivir. nl/publications/other/IViR_Recast_Final_Report_2006.pdf, p. 162. Consultado en octubre de 2015; Van Gompel, Stefan, Unlocking the Potential of Pre-Existing Content: How to Address the Issue of Orphan Works in Europe?, en http://www.ivir.nl/publicaties/vangompel/ IIC_2007_6_orphan_works.pdf, p. 3. Consultado en octubre de 2015; Conseil Supérieur de la Propriétée Litteraire et Artistique (cspla), Commission sur les euvres ORPHElines, Rapport, 19 marzo 2008, en http://www.cspla.culture.gouv.fr/CONTENu/
} 
titulares no pueden identificarse o localizarse ${ }^{2}$, ha aumentado recientemente sobre todo en el contexto de los proyectos de digitalización masiva que, basándose en el rápido desarrollo de las nuevas tecnologías, están orientados a la conservación y difusión de las creaciones intelectuales a través de los nuevos canales de distribución (p. ej., internet) ${ }^{3}$. En efecto, el amplio interés mostrado a nivel internacional por la tecnología digital ha dado lugar a diversos proyectos de digitalización de un gran número de obras protegidas por el derecho de autor que, a falta de una disciplina especial, generalmente pueden ser digitalizadas y puestas a disposición del público on line solamente con la autorización del autor (o titular de derechos). Sin embargo, el procedimiento orientado a identificar y localizar el autor o titular de derechos para obtener la autorización requerida (denominado en inglés right clearance) puede no solamente ser una tarea ardua y costosa, sino incluso imposible para un significativo número de obras ${ }^{4}$.

rapoeuvor08.pdf, p. 9. Consultado en octubre de 2015; Van Eechoud, Mireille, Orphan Works, en Harmonizing European Copyright Law: the Challenges of Better Law Making, La Haya, Kluwer Law International, 2009, p. 264 y sig.; Vuopala, Anna, Assessment of the Orphan Works Issue and Costs for Right Clearance, European Commission DG Information Society and Media, Unit E4 Access to Information, mayo 2010, en http://ec.europa.eu/ information_society/activities/digital_libraries/doc/reports_orphan/anna_report.pdf, p. 11 . Consultado en octubre de 2015; SAPPA, Cristiana, La propriété littéraire et artistique au service des institutions muséales à l'ère du numérique : analyse comparée en droit français et italien, Anrt, Lille, 2011, p. 271; kea European Affairs, Audiovisual Orphan Works in Europe - National survey, Report prepared for the W Study KEA, Report prepared for the British Film Institute, 2011, en http://www.keanet.eu/docs/OrPHAN\%2OWOrKs\%2OSTUDY\%20 KEA.pdf. Consultado en octubre de 2015.

2. Esta definición de obra huérfana, que se centra en la identificación y localización del autor y de los derechohabientes, es generalmente compartida por la doctrina. Véanse, entre otros, Huang, Olive, U.S. Copyright Office Orphan Works Inquiry: Finding Homes for the Orphans, en Berkeley Tech. L.J., 2006, p. 265; LANG, Orphan Works and the Google Book Search Settlement: An International Perspective, en N.Y. L. Sch. L. Rev., 2010-11, p. 116; Samuelson, Paula, The Google Books Search Settlement as Copyright Reform, en Wisc. L. Rev., 2011, p. 483; Hansen, David, Orphan Works: Definitional Issues, en Berkeley Digital Library Copyright Project, Berkeley Digital Library Copyright Project, White Paper No. 1, 2011, p. 4.

3. Al respecto véanse Lessig, Lawrence, Free culture: How Big Media Uses Technology and the Law to Lock Down Culture and Control Creativity, Penguin Press, New York, 2004, p. 288 sig.; Varian, Copyright Term Extension and Orphan Works, en Industrial and Corporate Change, 2006, en http://icc.oxfordjournals.org/content/15/6/965.full.pdf?keytype=ref\&ijkey =OrsmieeZhdWYKlJ, p. 968 s. Consultado en octubre de 2015; VAN Gompel, ob. cit., p. 7; Ivir, ob. cit., p. 161; Caso Roberto, I libri nella "tempesta perfetta": dal copyright al controllo delle informazioni digitali, en http://eprints.biblio.unitn.it/archive/4131. Consultado en octubre de 2015; SAPpa, ob. y loc. cit.

4. Un estudio reciente demostró que para un gran proyecto de digitalización de 500.000 fotografías y 5.000 películas llevado a cabo en los Países Bajos fue estimado un costo total de 625.000 euros para obtener las autorizaciones de los titulares de derechos y una duración del proceso de cuatro años; v. Vuopala Anna, Op. cit., p. 6.

En el Reino Unido una investigación sobre las colecciones de obras presentes en las organizaciones del sector público estimó que serían necesarias 6,5 millones de horas de 
Toda vez que es probable que quien emprenda un proyecto de digitalización masiva se encuentre frente a obras huérfanas, es claro que las obras huérfanas, o más bien, las reglas tradicionales del derecho de autor aplicables a las obras huérfanas, representan un problema que obstaculiza la realización de los mencionados proyectos 5 . El problema ha suscitado un amplio debate internacional que ha evidenciado la dimensión global del mismo y la gran cantidad de obras huérfanas existentes. Esto ha llevado a discutir sobre la necesidad de introducir nuevas reglas en el sistema de derecho de autor tradicional con el fin de combatir las principales causas de "orfandad" de una obra ${ }^{6}$. $\mathrm{Al}$ respecto, algunos doctrinantes de materias económicas han identificado el problema de las obras huérfanas como una particular hipótesis de "pérdida de mercado" (missing market) ${ }^{7}$. Una solución eficiente parece imposible a menos que el usuario que quiere digitalizar una obra y el titular de derechos entren en contacto y llegen a un acuerdo sobre las condiciones de utilización de la obra a digitalizar (precio, límite de la utilización, tiempo y otras condiciones) ${ }^{8}$. En el caso de las obras huérfanas no es posible llegar a dicho acuerdo, lo cual depende de causas externas a la lógica del intercambio y conduce a una lose-lose situation ${ }^{9}$ : el usuario potencial pierde la posibilidad de utilizar la obra y el autor pierde la oportunidad de obtener beneficios económicos

trabajo para encontrar los titulares de 13 millones de obras objeto de la investigación: Joint Information Systems Committee, jisc, In from the Cold: An assessment of the scope of "Orphan Works" and its impact on the delivery of services to the public, JISC Collections Trust, 2009, p. 21.

5. Sobre el tema v. Hansen, ob. cit., p. 10; Ginsburg, Jane, Contracts, Orphan Works, and Copyright Norms: What Role for Berne and TRIPs?, en Columbia Public Law and Legal Theory Working Papers, Paper 09162, 2009, en http://lsr.nellco.org/columbia_pllt/09162, p. 14. Consultado en octubre de 2015. Les Archives Françaises du Film, Orphan Works, en http://www.cnc-aff.fr/internet_cnc/Internet/ARemplir/Docs/Klimpel_en.pdf. Consultado en octubre de 2015; Commission of the European Communities, Communication from the Commission to the European Parliament, the Council, the European Economic and Social Committee and the Committee of the Regions, 'i2010 Digital Libraries', Сом(2005) 465 final, p. 5 ss.; Commission of the European Communities, Communication from the Commission to the European Parliament, the Council, the European Economic and Social Committee and the Committee of the Regions, A Digital Agenda for Europe, Consultado en octubre de 2015.

6. Sobre las causas de "orfandad" v. SAPpa, Cristiana, Opere orfane: la soluzione europea, en NLCC, 2014, p. 672.

7. Sobre el tema v. Farchy, Joelle y Petrou, Jessica, Optimizing use of orphan works while respecting intellectual property rights: A law and economics perspective, en Queen Mary Journal of Intellectual Property, 2012, p. 5; Urban, How Fair Use Can Help Solve the Orphan Works Problem, en Berkeley Tech. L.J., 2012, p. 21 ss.; KHONG, Orphan Works, Abandonware and the Missing Market for Copy righted Goods, en IJLEIT, 2007, p. 10 ss.

8. Farchy y Petrou, ob. y loc. cit.; Khong, ob. y loc. cit.; Loren, ob. cit., p. 22; Van Eechoud, ob. cit., p. 272 ss.; Katz, The orphans, the market, and the copyright dogma, en Berkeley Technology L.J., 2012, en http://papers.ssrn.com/sol3/papers.cfm?abstract_id=2118886, p. 4. Consultado en octubre de 2015.

9. Lifshitz-Goldberg, Orphan Works, Lecture Summary wipo Seminar, May, 2010, 
de los usos potenciales que se derivan de la digitalización, a causa de contingencias externas que limitan el acceso a millones de obras ${ }^{10}$.

Para superar este problema han sido propuestas diversas soluciones, entre las cuales se puede mencionar no solo el sistema de licencia colectiva ampliada adoptado en Dinamarca, que atribuye a una sociedad de gestión colectiva dotada de requisitos idóneos de representatividad el poder de estipular con los usuarios acuerdos económicos que incluyen obras huérfanas; o bien el establecimiento de un ente público para la concesión de licencias para utilizar obras huérfanas, como ocurre en Canadá; sino también otras hipótesis que aún no han sido adoptadas en la legislación de ciertos países, como por ejemplo la propuesta de limitar los mecanismos de tutela en los casos que se refieran a obras huérfanas, o la relativa a la necesidad de incrementar obligatoriamente la información relativa a los autores y sus obras ${ }^{11}$.

En Europa, teniendo como principal punto de referencia la creación de bibliotecas digitales, ante las distintas opciones para el establecimiento de un cuadro jurídico para la explotación de las obras huérfanas, la Directiva 2012/28/UE, aprobada el 4 de octubre de 2013, ha escogido el camino de excepciones al derecho de autor junto al reconocimiento recíproco de la condición de obra huérfana. Según el análisis de la Comisión Europea, una excepción al derecho de autor parece ser eficiente en cuanto no introduce modificaciones estructurales a los principios tradicionales del derecho de autor, no necesita que se efectúen licencias ni tampoco la creación de un ente que pueda conceder tales licencias, requiriendo solamente la realización de una búsqueda diligente por parte de las instituciones beneficiarias de la excepción ${ }^{12}$. En la dirección señalada por la Directiva, que ha introducido por primera vez una regulación orgánica sobre algunos usos permitidos de las obras huérfanas, se ha movido el legislador italiano definiendo el ámbito de aplicación de la excepción con referencia a parámetros objetivos y subjetivos.

en http://www.wipo.int/edocs/mdocs/sme/en/wipo_smes_ge_10/wipo_smes_ge_10_ref_theme11_02.pdf, p. 4 .

10. Copyright Office, Library of Congress, Orphan Works and Mass Digitization, Notice of Inquiry, October 12, 2012, p. 645-655.

11. Para una panorámica de las diferentes normativas en materia de obras huérfanas adoptadas en otros países véase, entre otros, Vetulani, Agnieszka, The Problem of Orphan Works in the EU, An overview of legislative solutions and main actions in this field, European Commission, DG Information Society and Media, Unit E4: Digital Libraries and Public Sector Information, 2008, en http://ec.europa.eu/information_society/activities/digital_libraries/doc/, p. 5. Consultado en octubre de 2015.

12. European Commission, Commission Staff Working Paper - Impact Assessment on the Cross-Border Online Access to Orphan Works Accompanying the Proposal for a Directive of the European Parliament and of the Council on Certain Permitted Uses of Orphan Works, COM (2011) 289 final, p. 37. Sobre el tema expresan una crítica Borghi y Karapapa, Copyright and Mass Digitization, A Cross-Jurisdictional Perspective, Oxford University Press, Oxford, 2013, p. 82. 


\section{I. Ámbito De APLICACiÓn DE LA NORMATIVA ITALIANA EN MATERia DE OBRAS HUÉRFANAS}

El Decreto legislativo 163 del 10 de noviembre de 2014 ha introducido el nuevo artículo 69 bis de la Ley italiana de derechos de autor para determinar cuáles son los sujetos que pueden utilizar obras huérfanas. Entre estos están comprendidos exclusivamente las bibliotecas, centros de enseñanza y museos, accesibles al público, así como los archivos, los organismos de conservación del patrimonio cinematográfico o sonoro y los organismos públicos de radiodifusión, siempre que las obras que hagan parte de las colecciones de tales instituciones culturales sean utilizadas con el fin de alcanzar objetivos relacionados con su misión de interés público ${ }^{13}$.

La delimitación por lo tanto es doble, ya que se caracteriza no solo por el tipo de sujeto sino además por la finalidad que este persigue. En efecto -como lo aclara también el vigésimo considerando de la Directiva-, la misión de interés público objeto de tutela es en particular la relativa a la conservación y restauración de las colecciones de las instituciones beneficiarias de la excepción, incluidas sus colecciones digitales, con fines culturales y educativos ${ }^{14}$. Quedan por lo tanto excluidos del ámbito de aplicación de la nueva normativa sobre obras huérfanas, no solo los sujetos privados distintos de las instituciones culturales antes mencionadas, sino además aquellas instituciones culturales que no actúen en función de un interés público ${ }^{15}$.

Pasando al ámbito objetivo, el nuevo artículo 69 ter señala que están comprendidas en la esfera de aplicación de la nueva normativa las obras protegidas por el derecho de autor y las creaciones objeto de derechos conexos, que hayan sido publicadas por primera vez en un Estado miembro de la Unión Europea, o a falta de publicación, cuya primera radiodifusión haya tenido lugar en un Estado miembro $^{16}$. El ámbito de aplicación comprende específicamente las obras publicadas en forma de libros, revistas, periódicos u "otras publicaciones", al igual que las obras cinematográficas o audivisuales y los fonogramas, siempre que sean conservados en las colecciones de bibliotecas, centros de enseñanza o museos accesibles al público, así como en las colecciones de archivos o de organismos de conservación del patrimonio cinematográfico o sonoro; también resultan incluidas las obras cinematográficas o audiovisuales y los fonogramas producidos por organismos de

13. Art. 69 bis, incisos 1 y 2, Ley 633/1941. Cfr. Considerando 1 y art. 1 de la Directiva 2012/28/UE.

14. Sobre la misión de interés público véase Giondano, Domenico, L’ambito di applicazione della Direttiva europea 2012/28 sulle orphan works, en AIDA, 2013, p. 130 ss.

15. Cfr. el 20. ${ }^{\circ}$ considerando de la Directiva 2012/28/Ue.

16. Art. 69 ter Ley 633/1941. Cfr. art. 1, par. 2, 3 y 4, Directiva 2012/28/ue. 
radiodifusión hasta el 31 de diciembre de $2002^{[17]}$ y que figuren en sus archivos. La nueva normativa se aplica además a las obras y prestaciones protegidas que estén insertadas o incorporadas en las obras o fonogramas ya mencionados ${ }^{18}$.

Por otro lado, quedan fuera del ámbito de aplicación de la Ley las obras huérfanas que se encuentren en el comercio ${ }^{19}$. Además, la normatividad se entiende sin perjuicio de las disposiciones existentes en materia de obras anónimas o seudónimas ${ }^{20}$.

\section{Búsqueda DILIGente}

Una vez delimitado el campo de aplicación, el legislador italiano establece las condiciones necesarias para que una obra pueda considerarse huérfana (art. 69 quater).

Debe tratarse de una obra (o fonograma) con respecto a la cual ninguno de los titulares de derechos haya sido identificado, o de estarlo, no haya sido localizado a pesar de haber sido efectuada una "búsqueda diligente" según determinadas modalidades ${ }^{21}$. Ahora bien, en caso de que exista más de un titular de derechos y no todos hayan sido identificados o, a pesar de haber sido identificados, no hayan sido localizados tras haber efectuado una búsqueda diligente, la obra podrá ser utilizada solo en los términos y límites de la autorización otorgada por parte de los titulares de derechos que hayan sido identificados y localizados ${ }^{22}$.

La atribución de la condición de obra huérfana, por lo tanto, es consecuencia de la realización de una búsqueda diligente por parte de las bibliotecas y demás organizaciones identificadas por el artículo $69 \mathrm{bis}^{23}$. Por tal motivo, ha sido introducida una normativa detallada sobre la búsqueda diligente que necesariamente debe preceder cualquier tipo de utilización de las obras que no haya sido autorizado por el autor.

17. El 10. ${ }^{\circ}$ considerando de la Direttiva sobre obras huérfanas explica la elección de dicha fecha: "Dada la especial situación de los organismos de radiodifusión como productores de fonogramas y material audiovisual, y la necesidad de adoptar medidas que limiten el fenómeno de las obras huérfanas en el futuro, resulta apropiado fijar una fecha límite para la aplicación de la presente Directiva a las obras y los fonogramas contenidos en los archivos de los organismos de radiodifusión”.

18. Art. 69 ter, inciso 3, Ley 633/1941. Cfr. art. 1, par. 4, Directiva 2012/28/ue.

19. Art. 69 quater, inciso 12, Ley 633/1941. Esto significa que solamente las obras huérfanas que están fuera del comercio pueden ser utilizadas por las instituciones beneficiarias de la excepción.

20. Art. 69 quater, inciso 13, Ley 633/1941.

21. Art. 69 quater, inciso 1, Ley 633/1941. Cfr. art. 2, par. 1, Directiva 2012/28/UE. Sobre el tema véase CARRARo GIUSEPPE, Le opere orfane nella prospettiva delle formalità costitutive e pubblicitarie, en AIDA, 2013, p. 107 ss.

22. Art. 69 quater, inciso 7, Ley 633/1941. Cfr. art. 2, par. 2, Directiva 2012/28/UE.

23. Cfr. art. 3 Directiva 2012/28/UE. 
El nivel de diligencia es establecido principalmente con referencia al examen de las "fuentes de información apropiadas". El legislador italiano ha determinado las "fuentes apropiadas" para cada categoría de obras, siguiendo las indicaciones mínimas fijadas por la Directiva, y ha previsto la consulta de las siguientes principales fuentes de información: el Registro Público General de Obras Protegidas que depende del Ministerio de Bienes, Actividades Culturales y Turismo, el depósito legal, las bases de datos de las sociedades de gestión colectiva y otras bases de datos y sistemas que integran múltiples bases de datos y registros, como por ejemplo $A R R O W^{24}$.

Más específicamente, el artículo 69 septies enumera las fuentes de información distinguiéndolas con base en el tipo de obra y señala, entre otras cosas, que (i) para los libros publicados será necesario consultar también el Sistema Bibliotecario Nacional, incluidos los ficheros de autoridades para los autores; (ii) para periódicos y revistas, el ISSN (International Serial Standard Number) y los índices y catálogos de los fondos y las colecciones de bibliotecas; (iii) para las obras audiovisuales y fonogramas, las asociaciones italianas de productores, los títulos de crédito y demás información que figure en el embalaje de la obra.

La nueva normativa prevé además que a través de un decreto del Ministerio de Bienes, Actividades Culturales y Turismo, previa consulta a las asociaciones más representativas de titulares de derechos y de usuarios, podrán ser identificadas otras fuentes de información que deberán ser tenidas en cuenta en el desarrollo de la búsqueda ${ }^{25}$. La búsqueda del autor y el procedimiento de consulta de las fuentes, además de ser efectuado con diligencia, deberá ser realizado según los principios de buena fe e integridad.

El legislador italiano tiene el objetivo de evitar la duplicidad de las búsquedas y para ello ha previsto que estas sean efectuadas al interior del Estado miembro de primera publicación de la obra, o a falta de publicación, en el Estado miembro donde haya tenido lugar la primera radiodifusión. Dicha regla tiene una excepción en cuanto a las obras cinematográficas y audiovisuales cuyo productor tenga su sede o residencia habitual en un Estado miembro; en tal caso la búsqueda diligente deberá llevarse a cabo en el Estado miembro de su sede o residencia habitual.

24. ARROW es un instrumento técnico orientado a facilitar la identificación y gestión de derechos de las obras publicadas en Europa y ha sido respaldado por la Comisión Europea que ha aprovado su financiamento con el fin de encontrar la forma de aclarar la condición de las obras huérfanas. El sucesor de ARROw es ARROwplus que trata de mejorar las funciones de ARROw: cfr. http://www.arrow-net.eu/what-arrow-plus. Consultado en octubre de 2015. Véase también Colin, Caroline, Registers, databases and orphan works, en Copyright and cultural heritage: preservation and access to works in a digital world, Edward Elgar, Cheltenham, 2010, p. 31.

25. Art. 69 quater, inciso 2, Ley 633/1941 
La normativa sobre la búsqueda diligente aclara además que es necesario que se mantenga la documentación relativa a la búsqueda efectuada y la información adquirida en el proceso de búsqueda, y que todo sea puesto a disposición del público $^{26}$. Las entidades beneficiarias de la nueva normativa no solo deben mantener la documentación que se refiere a los resultados de las búsquedas diligentes que han efectuado y que hayan llevado a la conclusión de que una obra o fonograma debe considerarse obra huérfana, sino que además deben entregar los mencionados resultados al Ministerio de Bienes, Actividades Culturales y Turismo, e incluir la información relacionada con el uso que las entidades hacen de las obras huérfanas y cualquier cambio en la condición de obra huérfana de las obras y los fonogramas que utilicen ${ }^{27}$. Por otro lado, durante la utilización de las obras, las organizaciones culturales beneficiarias deberán indicar el nombre del autor o de los titulares de derechos que hayan sido identificados ${ }^{28}$.

La información obtenida deberá ser registrada en una base de datos en línea única y accesible al público, lo que permitirá a las instituciones que efectúan búsquedas diligentes y a los titulares de derechos tener un fácil acceso a dicha información ${ }^{29}$.

\section{Usos AUTORIZADOS DE OBRAS HUÉRFANAS}

Con el fin de permitir a las entidades beneficiarias de la nueva normativa el uso de las obras húerfanas que hacen parte de sus colecciones, el legislador italiano, siguiendo los parámetros de la Directiva, introduce una excepción y limitación al derecho de reproducción y al derecho de puesta a disposición del público adicional a las excepciones y limitaciones ya existentes en la Ley de derechos de autor italiana.

En particular, los usos autorizados de las obras huérfanas consisten en la facultad de reproducir la obra a efectos de digitalización, puesta a disposición del público, indexación, catalogación, conservación o restauración ${ }^{30}$. Las entidades comprendidas en el ámbito subjetivo de la nueva normativa tienen además la facultad de poner a disposición del público la obra huérfana de tal forma que cualquier persona pueda acceder a ella desde el lugar y en el momento que elija, lo que permite a las instituciones culturales poner a disposición del público las copias de las obras huérfanas que hacen parte de sus colecciones ${ }^{31}$.

26. Art. 69 quater, incisos 14 y 15, Ley 633/1941 Cfr. art. 3, inciso 5, Dir. 2012/28/Ue.

27. Art. 69 quater, incisos 4 y 6, Ley 633/1941; cfr. art. 3, inciso 5, dir. 2012/28/Ue.

28. Art. 69 bis, inciso 4, Ley 633/1941; cfr. art. 6, inciso 3, dir. 2012/28/ue.

29. La Directiva sobre obras huérfanas ha previsto que dicha base de datos sea administrada e instituida ante la Oficina de armonización del mercado interior.

30. Art 69 bis, inciso 1, Ley 633/1941.

31. Cfr. Montagnani, Maria Lilla', Le utilizzazioni delle opere orfane, en AIDA, 2013, p. 162 ss. Sobre la facultad de meter a disposición de un público distante la obra véanse 
En principio los destinatarios de la nueva normativa no pueden utilizar las obras huérfanas para fines distintos al ejercicio de su misión de interés público, y pueden conservar, restaurar y facilitar el acceso de las obras y los fonogramas que figuren en su colección solo para "fines culturales y educativos" ${ }^{2}$. Por lo tanto, el ejercicio de la misión de interés público condiciona el ámbito de la excepción en comento ${ }^{33}$.

La nueva normativa autoriza a las instituciones culturales para llevar a cabo la explotación comercial de las obras huérfanas de manera limitada, es decir, a los efectos exclusivos de "cubrir los costos derivados de la digitalización de obras huérfanas y de su puesta a disposición del público" ${ }^{34}$. Las instituciones pueden también involucrar entidades del sector privado a través de acuerdos de asociación orientados a la valorización y aprovechamiento de las obras huérfanas, siempre dentro de los mencionados límites de utilización y en el ámbito del ejercicio de la misión de interés público ${ }^{35}$. En consecuencia, los "socios" privados no podrán utilizar directamente las obras huérfanas, ni controlar su utilización o imponer restricciones a la utilización de las obras por parte de las instituciones culturales beneficiarias de la normativa ${ }^{36}$. Su participación parece reducirse solamente al patrocinio pro bono o a la donación de fondos ${ }^{37}$.

\section{LA COMPENSACIÓN EQUITATIVA PARA LOS TITULARES DE DERECHOS}

Las nuevas disposiciones introducidas por el Decreto legislativo 163 de 2014 incluyen también la regulación de las situaciones en que se ponga fin a la condición de obra huérfana. En efecto, el nuevo artículo 69 sexies de la Ley italiana de derechos de autor prevé que el titular de derechos de una obra o fonograma considerados huérfanos tiene la posibilidad de poner fin a la condición de orfandad en

Ricolfi, Marco, Diritto Industriale, en Trattato di Diritto Commerciale, Padova, 2001, p. 440 ss.; Auteri, Paolo, Diritto di autore, Diritto industriale, Torino, 2009, p. 616.

32. Art 69 bis, inciso 2, Ley 633/1941; cfr. art. 6, par. 3, dir. 2012/28/ue.

33. Al respecto Pollaud-Dulian, Utilisations autorisées des auvres orphelines, en RTD Com., 2012, p. 782.

34. Art 69 bis, inciso 3, Ley 633/1941; cfr. art. 6, par. 2, Dir. 2012/28/ue.

35. Art 69 bis, inciso 5 Ley 633/1941; cfr. 21 y 22 considerando dir. 2012/28/ue.

36. Los acuerdos no pueden ser contrarios a la regla de los tres pasos: art 69 bis, inciso 5, y cfr. 22. ${ }^{\circ}$ considerando Dir. 2012/28/UE.

37. Sobre el tema véase Information Sans Frontières, Response to the Final Compromise Text of the Orphan Works Directive, 22 giugno 2012, en http://informationsansfrontieres. eu/2012/06/22/a-response-from-information-sans-frontieres-to-the-final-compromise -text-of-the-orphanworks-directive-4/, p. 2. 
cualquier momento ${ }^{38}$. Así mismo, el titular de derechos puede pedir en cualquier momento que se le otorgue una compensación equitativa por el uso de su obra ${ }^{39}$.

En caso de que un titular de derechos manifieste su voluntad de poner fin a la condición de orfandad, será preciso que las instituciones beneficiarias de la nueva excepción, a pesar de haber efectuado una búsqueda diligente, procedan al pago de la compensación equitativa por cualquier utilización de la obra realizada hasta la fecha. Por otro lado, las utilizaciones de obras huérfanas en curso de ejecución no podrán continuar sin el consentimiento del titular de derechos que ha reaparecido. El autor o titular de derechos podría entonces ejercitar sus facultades impidiendo que continúe la utilización de la obra ${ }^{40}$.

La compensación puede ser establecida mediante acuerdos entre las asociaciones representativas de titulares de derechos y las instituciones beneficiarias de la excepción objeto de estudio, y deberán tener en cuenta los objetivos de promoción cultural relacionados con la utilización de la obra, la naturaleza no comercial del uso realizado por los beneficiarios de la excepción en conexión con su misión de interés público, al igual que el eventual daño causado a los titulares de derecho ${ }^{41}$.

\section{Críticas a la normativa italiana en materia DE OBRAS HUÉRFANAS}

Las normas antes descritas presentan diversos aspectos críticos que corresponden a los que ya han sido evidenciados por algunos doctrinantes que han comentado la Directiva sobre obras huérfanas ${ }^{42}$. En primer lugar existen críticas en lo que respecta a los sujetos beneficiarios de la excepción. Se ha sostenido que la utilización de obras huérfanas por parte de entes privados o distintos a las instituciones culturales podría facilitar y acelerar los procesos de digitalización masiva gracias a los recursos que tales sujetos estarían en condiciones de invertir con el fin de obtener beneficios comerciales de la utilización de obras huérfanas ${ }^{43}$.

38. Art. 69 quinquies, inciso 1, Ley 633/1941; cfr. art. 5 dir. 2012/28/uE.

39. Art. 69 quinquies, inciso 2, Ley 633/1941; cfr. art. 6, par. 5, y 18 considerando dir. 2012/28/ue.

40. Romano, Attribuzione e revoca dello status di opera orfana, ovvero nemesi dell'iperprotezionismo, en AIDA, 2013, p. 158; véase también Torremans, Ginsburg, Ficsor, Rosen, Bouchard, Walter y Marzano, Orphan Works: Compatibility of the Draft Directive with the International Norms, 21 de mayo de 2012, en http://www.copyrightseesaw.net/archive/?sw_10_ item $=21$, p. 2 ss. Consultado en octubre de 2015.

41. Art. 69 quinquies, inciso 3, Ley 633/1941; cfr. $18 .^{\circ}$ considerando dir. 2012/28/UE. Véanse además Hansen, Hashimoto, Hinze, Samuelson y Urban, Solving the Orphan Works Problem for the United States, en Columbia Journal of Law \& the Arts, 2013, p. 39.

42. Al respecto véase Rosati, The Orphan Works Directive, or throwing a stone and hiding the hand, in JIPLP, 2013, p. 309.

43. Cfr. Borghi e Karapapa, ob. cit., p. 86; Rosati, ob. y loc. cit. 
Ahora bien, los acuerdos de asociación público-privada establecidos en la Directiva y en consecuencia previstos también por el legislador italiano resultan limitados, toda vez que, si bien los socios privados pueden financiar y facilitar la digitalización o la puesta a disposición al público de las obras huérfanas mediante acuerdos con las instituciones culturales, los entes privados no pueden utilizar las obras o controlar su utilización. Tal limitación podría disuadir la participación de estos sujetos (como Google), que suelen contribuir a la digitalización de las obras, incluso con importantes recursos, siempre que exista la posibilidad de poner sucesivamente las obras a disposición del público por medio de motores de búsqueda o a través de plataformas de intercambio para obtener un beneficio comercial ${ }^{44}$.

Cabe aclarar que la delimitación de los sujetos y la exclusión de los usos autorizados por parte de sujetos privados (aun cuando "socios" de las instituciones culturales en los proyectos de digitalización), además de estar en consonancia con las iniciativas y estudios efectuados por la Comisión Europea antes de la Directiva que habían evidenciado los posibles perjuicios potenciales para los titulares de derechos que se ocasionarían al permitir la utilización de obras huérfanas por parte de operadores comerciales, resulta justificada por razones de carácter sistemático.

La reducida extensión del ámbito subjetivo, en efecto, parece impuesta por la necesidad de respetar la regla de los tres pasos ${ }^{45}$. La regla de los tres pasos ha sido prevista en el Convenio de Berna para la Protección de las Obras Literarias y Artísticas y en otros convenios internacionales (al igual que en la Directiva 2001/29/CE). De conformidad con esta regla las excepciones y limitaciones al derecho de autor pueden aplicarse solamente en (i) determinados casos especiales, (ii) que no atenten contra la explotación normal de la obra (iii) ni causen un perjuicio injustificado a los intereses legítimos del autor. Toda vez que la reforma para autorizar la utilización de algunas obras huérfanas ha introducido una nueva excepción o limitación al derecho de autor, esta excepción está sujeta a la ya mencionada regla.

En ese orden de ideas, mientras la utilización de ciertas obras huérfanas por parte de algunos sujetos (instituciones culturales públicas) parece conforme al primer paso de la regla, se ha señalado que la expansión de los beneficiarios amparados por la nueva normativa habría llevado la excepción fuera de los límites relativos a "determinados casos especiales" ${ }^{3}$. La extensión del ámbito subjetivo de la nueva normativa, por ende, no puede ser efectuada a nivel interpretativo sino que necesitaría que sea reconsiderado el instrumento adoptado para autorizar la utilización de obras huéfanas.

44. Sobre el tema v. MAnsani, Proprietà intellettuale e giacimenti culturali, en AIDA, Milano, 2013, p. 117 ss.

45. Torremans, Ginsburg, Ficsor, Rosen, Bouchard, Walter y Marzano, ob. y loc. cit. 46. Ibíd. 
En lo que respecta al ámbito objetivo, se debe señalar que muchas obras que representan una parte importante del patrimonio cultural existente siguen estando fuera del campo de aplicación de la normativa ${ }^{47}$.

En primer lugar, las fotografías no fueron tomadas en consideración por la Directiva y en consecuencia tampoco por el legislador italiano; esta decisión ha sido calificada por la doctrina como poco comprensible toda vez que las fotografías constituyen una parte importante del patrimonio cultural de las bibliotecas, centros de enseñanza y museos ${ }^{48}$. Solamente las fotografías que estén insertadas o incorporadas en las obras ennumeradas por la Ley cumplen con el perfil objetivo requerido. Esto parecería comportar una división poco coherente en materia de fotografías: por un lado se tienen las fotografías "huérfanas" que hayan sido incorporadas en las obras mencionadas por la Ley, las cuales podrán ser utilizadas, y por otro, las fotografías que no han sido incorporadas en otras obras, las cuales quedan por fuera del ámbito de aplicación de la Ley y por ende no pueden ser utilizadas ${ }^{49}$.

La nueva normativa tampoco comprende las obras anónimas o seudónimas respecto a las cuales se mantienen las disposiciones nacionales existentes ${ }^{50}$. Posiblemente la exclusión resulte justificada por el hecho de que las obras anónimas o seudónimas no son obras "abandonadas" a su destino y la elección del autor de no aparecer mencionado directamente puede ser fruto de un acuerdo con el editor $^{51}$. En cambio, en materia de obras huérfanas, toda vez que el autor no puede identificarse, no se pueden conocer ni siquiera las condiciones de utilización de la obra. Sin embargo, puesto que también en el caso de las obras anónimas puede suceder que no sea identificado o localizado por lo menos el intermediario y en consecuencia no sea posible conocer la condiciones de utilización que deseaba incluir el autor, no resulta comprensible la ratio de la exclusión ${ }^{52}$.

Por otro lado, existen algunas utilizaciones de obras huérfanas que a pesar de no ser mencionadas por la Directiva y por lo tanto no estar incluidas en la normativa italiana, resultan fundamentales en materia de investigación científica o de mercado. Es el caso de las actividades de data o text mining, que tienen por

47. Cfr. Giordano, ob. cit., p. 135; mansani, Proprietà intellettuale e giacimenti culturali, en AIDA, Milano, 2013, p. 117. En materia de patrimonios de empresas y obras huérfanas véase, De Angelis, Daniele, Il copyright tra opere orfane e patrimoni aziendali ignoti, 2014, en http://www.diritto24.ilsole24ore.com/art/avvocatoAffari/mercatiImpresa/2014-11-17/ilcopyright-opere-orfane-e-patrimoni-aziendali-ignoti-142449.php.

48. Sappa, ob. ult. cit.; Giordano, ob. y loc. cit.

49. Sobre el tema véase Rodriguez, Juan Carlos, Libros huérfanos en libre disposición, en Delibros, 2012, en http://www.delibros.com/wp-content/uploads/2012/12/08Dossier-Dic.pdf, p. 20.

50. Art. 69 quater, inciso 13, Ley 633/1941; cfr. art. 2, par. 5, dir. 2012/28/ue.

51. Romano Rosaria, ob. cit., p. 153 ss.

52. Cfr. Giordano o, ob. cit., p. 137 ss. 
objeto la extracción de información de grandes conjuntos de datos, para lo cual generalmente es necesaria una reproducción de las obras ${ }^{53}$. $\mathrm{Al}$ respecto, teniendo en cuenta que el text y el data mining no fueron expresamente incluidos entre las utilizaciones autorizadas de obras huérfanas, se ha señalado que la nueva normativa habría podido otorgar la oportunidad para consentir expresamente a las instituciones culturales el desarrollo de dichas actividades ${ }^{54}$.

En lo que respecta al requisito de búsqueda diligente, parece razonable poner en duda que esta esté al alcance de todas las organizaciones beneficiarias de la excepción, ya que podría requerir que ellas cuenten con personal con conocimientos específicos en materia de derecho de autor capaz de identificar los titulares de derechos a menudo numerosos (piénsese en el caso de las obras complejas).

Además, incluso una búsqueda diligente podría fácilmente resultar no exhaustiva ni concluyente, a falta de sistemas de registro obligatorio de obras protegidas por el derecho de autor en registros públicos o en otros sistemas tomados en consideración por el legislador italiano como posibles fuentes de obtención de información (p. ej., el Sistema Bibliotecario Nacional, el issn -International Serial Standard Number-, el Registro Público General de Obras Protegidas y las bases de datos de las sociedades de gestión colectiva) ${ }^{55}$.

Por otro lado, la doctrina ha observado que ante la falta de una autoridad que controle el respeto de las normas de búsqueda diligente, la solución introducida puede resultar ineficaz ya que las instituciones culturales deberán simplemente autoevaluar si sus esfuerzos de búsqueda han sido suficientes y conservar la respectiva documentación para demostrarlo en caso de controversia. Es posible pensar que las modalidades de "autoevaluación" varíen entre las diferentes organizaciones culturales ${ }^{56}$.

Finalmente, los costos de la búsqueda y de conservación de la información correrán por cuenta de las organizaciones destinatarias de la excepción, lo que no es económicamente factible para muchas de ellas ${ }^{57}$. Por consiguiente, los requisitos de la búsqueda diligente impuestos por el legislador italiano, si bien resultan acordes con lo previsto en la Directiva, no están en consonancia con los proyectos de digitalización masiva de las colecciones de las instituciones culturales. La complejidad y los costos para llevar a cabo una búsqueda diligente para cada obra

53. Sobre el tema véase Witten y Frank, Data Mining: Practical Machine Learning Tools and Techniques, Elsevier, San Francisco, 2005.

54. Cfr. Borghi y Karapapa, ob. cit., p. 69 ss.

55. Romano, R., ob. cit., p. 149 ss.

56. Sobre el tema v. Schulze, ob. cit., p. 317.

57. Lauriat, Semi-Formal Copyright? The Past and Future of Orphan Works, en Art, Antiquity and Law, December 2012, en: http://ssrn.com/abstract=2238473 
huérfana pueden resultar muy altos y reducir las ventajas de la solución y de los incentivos para la utilización de las obras huérfanas ${ }^{58}$.

Otras críticas han sido formuladas en relación con la compensación equitativa destinada a los titulares de derechos. Estos, una vez que se hacen presentes, no pueden negar su autorización para las utilizaciones ya efectuadas de sus obras consideradas huérfanas, sino que reciben una remuneración razonable. A pesar de que el pago de la compensación equitativa se origina en la necesidad de encontrar un punto de equilibrio entre los intereses de los usuarios y los de los autores y titulares de derechos ${ }^{59}$, y comporta una degradación del derecho exclusivo sobre las obras que se reduce a un derecho a la "compensación equitativa", algunas instituciones culturales han manifestado una serie de preocupaciones respecto de tal obligación de pago ${ }^{60}$.

En primer lugar, la obligación es fuente de incertidumbre desde un punto de vista económico ya que no es fácil prever el monto del desembolso futuro. A la incertidumbre económica se suma la incertidumbre sobre la posible utilización futura de las obras huérfanas, toda vez que el autor (o titular de derechos) una vez reparezca podría objetar la utilización sucesiva de su obra. Esta situación no tiene previsto al menos un límite temporal como lo señalaba en cambio la propuesta original de Directiva de la Comisión Europea, según la cual el transcurso de un determinado término sin que el titular de derechos reaparezca habría eliminado cualquier obligación de pago ${ }^{61}$.

En síntesis, la nueva normativa parece imponer a las instituciones culturales un riesgo de pago de difícil cuantificación por los eventuales requerimientos de los titulares de derechos que pueden ser presentados en cualquier momento ${ }^{62}$,

58. Sobre el tema v. Ringnalda, Allard, Orphan Works, Mass Rights Clearance and Online Libraries: The Flaws of the Draft Orphan Works Directive and Extended Collective Licensing as a Solution, en Medien und Recht International (MR-Int), 2011, p. 7; URBAN, How Fair Use Can Help Solve the Orphan Works Problem, en Berkeley Tech. L.J., 2012, p. 1379; Van-Gompel, S., The Orphan Works Chimera and How to Defeat It: A View From the Atlantic, en Berkeley Tech. L.J., 2012, p. 1360; Hargreaves y Hugenholtz, Copyright Reform for Growth and Jobs: Lisbon Council Policy Brief, 2013, en http://www.ivir.nl/publications/hugenholtz/LisbonCouncil_policybrief.pdf, pp. 9 e 10.

59. Al respecto v. Cogo, Alessandro, La determinazione amministrativa dei corrispettivi, en AIDA, 2012, p. 193. Cfr. también Bertani, Michelle, Impresa culturale e diritti esclusivi, Milano, 2000, p. 464; Nivarra, L'equo compenso degli autori, en AIDA, 2005, p. 121; Giordan, ob. y loc. cit.

60. Information Sans Frontières, ob. cit., p. 2.

61. El término no podía ser menor a cinco años a partir de la fecha del acto que había dado origen a la solicitud: cfr. art. art. 7, par. 1, punto 5, propuesta de directiva sobre algunos usos autorizados de las obras huérfanas.

62. TransAtlantic Consumer Dialogue, Orphans Left Out in Cold: Final Vote on Weak Directive, IP Policy Blog, 12 de septiembre de 2012, en: http://tacd-ip.org/archives/742. Consultado en octubre de 2015. 
situación que podría disuadir a los destinatarios de la excepción de digitalizar sus colecciones ${ }^{63}$.

\section{Conclusiones}

La solución seguida por el legislador italiano en atención a las directrices señaladas por la Directiva 2012/28/UE no parece constituir un sólido punto de referencia para los proyectos de digitalización masiva ya que se trata de una solución limitada y parcial al problema de las obras huérfanas.

La nueva normativa, que introduce una excepción o limitación al derecho de autor y autoriza algunas utilizaciones de las obras huérfanas por parte de determinadas organizaciones, idealmente debería permitir al menos a las instituciones culturales emprender la digitalización a gran escala de obras huérfanas para ponerlas a disposición del público en internet con fines educativos y culturales.

Sin embargo, dicha finalidad (ya limitada desde un punto de vista subjetivo) parece poder llevarse a cabo solo en parte. En efecto, muchas obras huérfanas que constituyen una parte importante del patrimonio cultural existente en las instituciones culturales quedan fuera del ámbito objetivo de aplicación de la nueva normativa, como ocurre con las fotografías huérfanas y las obras anónimas huérfanas.

Por otro lado, en cuanto respeta al ámbito subjetivo, el legislador europeo habría podido ampliar los destinatarios de la excepción para incluir entes diversos de las instituciones culturales (y tal vez también sujetos privados), ya que la participación de tales sujetos posiblemente facilitaría los procesos de digitalización, sobre todo en el caso de entes dispuestos a invertir y poner a disposición recursos asumiendo su propio riesgo con la finalidad de obtener una ventaja comercial de la utilización de obras huérfanas.

A esto se añade que las instituciones culturales destinatarias de la normativa no pueden utilizar las obras huérfanas para fines diferentes a la misión de interés público. Pueden conservar, restaurar y mantener a disposición del público las obras y fonogramas que hacen parte de sus propias colecciones solamente para "fines culturales y educativos”. El desarrollo de la misión de interés público condiciona el ámbito de la excepción.

Los límites objetivos y subjetivos de la solución italiana y europea conducen a señalar que se trata de una solución parcial al problema. Como lo ha discutido la doctrina a nivel internacional, la solución al problema de las obras huérfanas tal vez requiere un compromiso a nivel internacional, ya que pensar en una respuesta limitada al territorio de un país ignoraría la principal característica de los proyectos de digitalización que se desarrollan a través de los nuevos canales de 
difusión, tales como internet, y que comportan una difusión global de las obras. El compromiso a nivel internacional, sin embargo, aún está muy lejos de alcanzarse.

\section{Bibliografía}

Auteri, Paolo, Internet ed il contenuto del diritto d'autore, en AIDA, 1996, p. 86. Auteri, Paolo, Protezione delle opere letterarie ed artistiche, commento al dpr. 8.1.1979 n. 19, en NLCC, 1980, p. 148.

Carraro, Giuseppe, Le opere orfane nella prospettiva delle formalità costitutive e pubblicitarie, en AIDA, 2013, p. 107.

Cogo, Alessandro, La determinazione amministrativa dei corrispettivi, en AIDA, 2012, p. 173.

De la Durantaye, Katharina, H Is For Harmonization: The Google Book Search Settlement and Orphan Works Legislation in the European Union, en New York Law School Law Review 55, n.o 1 2010/2011, p. 157.

Fabiani Mario, Opere orfane e diritti orfani, en IDA, 2009, p. 225.

Farchy, Joelle y Petrou, Jessica, Optimizing use of orphan works while respecting intellectual property rights: a law and economics perspective, en Queen Mary Journal of Intellectual Property, 2012, p. 250.

Galli, PaOlo, Archivi, banche dati ed opere o prodotti culturali orfani, en TorRe, Le carte e le immagini, Ediesse-Aamod, Roma, 2008, p. 227.

Giordano, Domenico, L'ambito di applicazione della Direttiva europea 2012/28 sulle orphan works, en AIDA, 2013, p. 130.

Hansen, David, Orphan Works: Causes of the Problem, en Berkeley Digital Library Copyright Project, White Paper No. 3, 2012.

Iglesias, María, Obras digitales y obras cautivas, 1st communia Conference on the Digital Public Domain, Louvain-la-Neuve (Belgio), 2008, en: http://www. communia-project.eu/node/110, Consultado en octubre de 2015.

Katz, Ariel, The orphans, the market, and the copyright dogma, en Berkeley Tech. L. J., 2012, p. 1285.

Khong, Olive, Orphan Works, Abandonware and the Missing Market for Copy Righted Goods, en IJLEIT 2007, p. 54.

Loren, Lydia, Abandoning the Orphans: An Open Access Approach to Hostage Works, en Berkeley Tech. L.J., 2012, p. 1431.

Montagnani, Maria Lilla', Le utilizzazioni delle opere orfane, en AIDA, 2013, p. 162.

Ricolfi, Marco, Copyright Policy for digital libraries in the context of the i2010 strategy, 1st communia Conference on the Digital Public Domain, Louvainla-Neuve (Belgio), 2008, en: http://www.communia-project.eu/node/110. Consultado en octubre de 2015. 
Ricolfi, Marco, Making Copyright Fit for the Digital Agenda, 12th eipin Congress 2011 - Constructing European IP: Achievements and new Perspectives, European Parliament, Strasburgo 2011, en: http://www.communia-project. eu/node/110. Consultado en octubre de 2015.

Ringnalda, Allard, Orphan Works, Mass Rights Clearance and Online Libraries: The Flaws of the Draft Orphan Works Directive and Extended Collective Licensing as a Solution, en Medien und Recht International (MR-Int), 2011, p. 3.

Romano, Rosaria, Attribuzione e revoca dello status di opera orfana ovvero nemesi delliperprotezionismo, en AIDA, 2013, p. 146.

Rosati, Eleonora, The Orphan Works Directive, or throwing a stone and hiding the hand, en JIPLP 2013, p. 303.

SAPPA, Cristiana, La soluzione europea al problema delle opere orfane, en NLCC, 2014, p. 672.

Sappa, Cristiana, La propriété littéraire et artistique au service des institutions muséales à l'ère du numérique analyse comparée en droit français et italien, ANRT, Lille, 2011.

SARTI, Davide, Gestione collettiva e modelli associativi, en aa.vv., Studi in onore di Gerhard Schricker, Giuffré, Milano, 2005.

Sarti, Davide, La "privatizzazione" dell'attività della SIAE. Diritto d'autore, reti telematiche e libere utilizzazioni per scopi didattici, en NLCC, 2009, p. 349.

UbertazZi, Luigi Carlo, Diritti d'autore e connessi. Scritti, Milán, Giuffrè, 2005.

Van Gompel, Stefan, Unlocking the Potential of Pre-Existing Content: How to Address the Issue of Orphan Works in Europe?, en IIC 2007, 669, en http://www. ivir.nl/publicaties/vangompel/IIC_2007_6_orphan_works.pdf

Vetulani, Agnieszka, The Problem of Orphan Works in the EU, An overview of legislative solutions and main actions in this field, European Commission, DG Information Society and Media, Unit E4: Digital Libraries and Public Sector Information, 2008, en http://ec.europa.eu/information_society/activities/ digital_libraries/doc/ reports_orphan/report_orphan_v2.pdf Consultado en octubre de 2015. 